Peer Reviewed Paper openaccess

\title{
Near infrared hyperspectral imaging of blends of conventional and waxy hard wheats
}

\author{
Stephen R. Delwiche, ${ }^{a, *}$ Jianwei Qin, ${ }^{b}$ Robert A. Graybosch, ${ }^{c}$ Steven R. Rausch ${ }^{\text {d }}$ and Moon S. Kim ${ }^{e}$ \\ aUSDA-ARS, Beltsville Agricultural Research Center, Food Quality Laboratory, Building 303, BARC-East, Beltsville, Maryland, 20705-2350, USA. \\ E-mail: stephen.delwiche@ars.usda.gov. ORCID: https://orcid.org/0000-0002-7533-9012 \\ bUSDA-ARS, Beltsville Agricultural Research Center, Environmental and Microbial Food Safety Laboratory, Building 303, BARC-East, Beltsville, \\ Maryland, 20705-2350, USA. ORCID: https://orcid.org/0000-0002-8061-4373 \\ CUSDA-ARS, Department of Agronomy, University of Nebraska, Lincoln, Nebraska, 68583, USA \\ ¿USDA-ARS, Beltsville Agricultural Research Center, Food Quality Laboratory, Building 303, BARC-East, Beltsville, Maryland, 20705-2350, USA. \\ ORCID: https://orcid.org/0000-0002-5501-5168 \\ eUSDA-ARS, Beltsville Agricultural Research Center, Environmental and Microbial Food Safety Laboratory, Building 303, BARC-East, Beltsville, \\ Maryland, 20705-2350, USA
}

\begin{abstract}
Recent development of hard winter waxy (amylose-free) wheat adapted to the North American climate has prompted the quest to find a rapid method that will determine mixture levels of conventional wheat in lots of identity preserved waxy wheat. Previous work documented the use of conventional near infrared (NIR) reflectance spectroscopy to determine the mixture level of conventional wheat in waxy wheat, with an examined range, through binary sample mixture preparation, of 0-100\% (weight conventional / weight total). The current study examines the ability of NIR hyperspectral imaging of intact kernels to determine mixture levels. Twenty-nine mixtures $(0,1,2,3,4,5,10,15, \ldots, 95,96,97,98,99,100 \%)$ were formed from known genotypes of waxy and conventional wheat. Two-class partial least squares discriminant analysis (PLSDA) and statistical pattern recognition classifier models were developed for identifying each kernel in the images as conventional or waxy. Along with these approaches, conventional PLS1 regression modelling was performed on means of kernel spectra within each mixture test sample. Results indicated close agreement between all three approaches, with standard errors of prediction for the better preprocess transformations (PLSDA models) or better classifiers (pattern recognition models) of approximately 9 percentage units. Although such error rates were slightly greater than ones previously published using non-imaging NIR analysis of bulk whole kernel wheat and wheat meal, the HSI technique offers an advantage of its potential use in sorting operations.
\end{abstract}

Keywords: wheat, hyperspectral imaging, mixture, waxy, amylose

\section{Introduction}

Waxy starches in cereal grains arise from the near complete absence of the linear chain $[\alpha-(1 \rightarrow 4)$-linked glucan units] macromolecule amylose, thus causing the starch to be composed exclusively of the branched $[\alpha-(1 \rightarrow 6)$-linkages] chain macromolecule amylopectin. In hexaploid wheat (Triticum aestivum L.), the waxy condition arises from the simultaneous presence of null mutations at each of the three genetic loci $\left(W_{x}-A 1, W_{x}-B 1\right.$ and $\left.W_{x}-D 1\right)$ that

Mention of trade names or commercial products is solely for the purpose of providing specific information and does not imply endorsement or recommendation by the USDA.

\author{
Correspondence \\ Received: 1 June 2017 \\ Revised: 24 October 2017 \\ Accepted: 24 October 2017 \\ Publication: 9 February 2018 \\ doi: $10.1255 /$ jsi.2018.a2 \\ ISSN: 2040-4565
}

Stephen Delwiche (stephen.delwiche@ars.usda.gov)

\author{
Citation \\ S.R. Delwiche et al., "Near infrared hyperspectral imaging of blends \\ of conventional and waxy hard wheats", J. Spectral Imaging 7, a2 (2018). \\ https://doi.org/10.1255/jsi.2018.a2 \\ (c) 2018 The Authors
}

This licence permits you to use, share, copy and redistribute the paper in any medium or any format provided that a full citation to the original paper in this journal is given. 
encode for the enzyme granule bound starch synthase (GBSS) which controls amylose synthesis. Starting with the development of waxy wheat genotypes in Japan, ${ }^{1}$ wheat breeding programmes in other regions have developed geographically adapted waxy wheats, including those in the US northern Great Plains. ${ }^{2}$ The reason for such breeding efforts has been the possibility that well-adapted, amylose-free or low-amylose wheat possesses unique starch processing properties that are independent of the usual protein dominated functional characteristics. ${ }^{3}$ Such wheats may offer unique uses for shelf life extension, ethanol production and non-food applications. ${ }^{4-6}$

From the perspective of commodity trade, the higher value for waxy wheat and the consequential need to keep it identity preserved require a rapid method for inspection of lots to verify pureness. Visual inspection is difficult due to the similarity in colour, size and shape of waxy and conventional wheat. Although genotyping can be performed by PCR, ${ }^{7-9}$ by protein analysis of the GBSS isoforms by ELISA ${ }^{10}$ or even by iodine staining and counting kernels, ${ }^{11}$ such procedures are not amenable to rapid analysis or feasible at commodity sales points. As an alternative, the authors have studied the use of conventional near infrared (NIR) spectroscopy for distinguishing waxy wheat from conventional wheat. ${ }^{12-14}$ More recent studies have examined the ability of NIR reflectance spectroscopy of bulk wheat or wheat meal to estimate the percentage by weight of conventional seed in mixtures of waxy and conventional wheat, ${ }^{15,16}$ with standard errors typically ranging from $4 \%$ to $9 \%$. With the vibrational frequencies of carbohydrate backbone structure outside the NIR region, the challenges to the NIR approach stem from chemical similarities of amylopectin and amylose molecules, thus leaving the possibilities of measurable differences to be from hydrogen-bonding effects or the presence of an amylose-lipid complex. Traditionally, the NIR method involves collecting spectra on bulk kernels or ground meal and, in either case, this means a composite of hundreds. Alternatively, hyperspectral imaging (HSI) offers the ability to develop spectrally based models at the individual kernel level, while doing so on all kernels within the hypercube image. To date, published work involving HSI analysis of constituents in cereals is limited, with studies focused on macro constituent analysis such as protein, oil and starch in maize, ${ }^{17}$ hardness in wheat ${ }^{18}$ or classification of waxy maize varieties. ${ }^{19}$ In the authors' previous research on wheat endosperm amylose concentration by $\mathrm{NIR},{ }^{14}$ it was suggested that changes in the strengths of the $\mathrm{C}-\mathrm{H}$ bonds and the hydrogen bonds, as affected by the differing degrees of crystallinity between amylopectin (more crystalline) and amylose (less crystalline), are responsible for their spectral differences. Additionally, differences in the prevalence of the amylose-lipid complex, as described for diploid cereal species $^{20}$ and later for hexaploid wheat, ${ }^{21}$ may also produce spectral differences. The intention of revisiting the topic of spectral measurement of the waxy wheat condition was to determine whether such techniques are applicable at the single kernel level through spectral imaging as the first step in the eventual design of a system that would use such imaging for sorting of kernels by their amylose concentration. Hence, the current study's objective was to examine the potential of HSI to measure mixture levels of conventional and waxy wheat genotypes using several established chemometric and statistical pattern recognition techniques. The samples used in this study were the same as used in an earlier study on conventional and waxy wheat mixture measurement, ${ }^{15}$ thus allowing for the direct comparison of results between traditional NIR reflectance spectroscopy and HSI.

\section{Materials and methods Wheat samples}

Hard wheat varieties or advanced breeding lines from Nebraska-grown 2011 and 2012 breeders' grow out trials have been previously described. ${ }^{15}$ Briefly, genotypes of wheat of the wild type condition for starch were paired with an equal number of fully waxy field samples (Table 1). Because of the limited supply of waxy field samples, an effort was made to select conventional samples that were close in protein content to their waxy counterparts and originated from the same geographical location. Additionally, with two of the waxy field samples being of sufficient quantity for additional pairings, conventional counterparts were selected to bracket the protein content of each field sample. Altogether, eight pairings were formed. The parent samples, with each in its own canvas bag, were placed in a $50 \mathrm{~L}$ polyethylene sealed container for a one-month moisture equilibration period, whereupon mixture laboratory samples (hereafter called test samples) for each pair were prepared by weighing out precisely defined portions to the nearest kernel. The equilibration period was imposed to minimise any moisture variation between parent samples as the causative effect on mixture prediction in NIR model- 
Table 1. Description of sample pairs (from Reference 15).

\begin{tabular}{|l|l|l|l|l|l|l|l|}
\hline & \multicolumn{2}{|c|}{ Variety or line } & & \multicolumn{2}{c|}{ Protein content (\% wb) } & \multicolumn{2}{c|}{ Total lipid content $(\%$ wb) } \\
\hline Pair & Waxy & Conventional & Year & Waxy & Conventional & Waxy & Conventional \\
\hline A & NX1OMD 2216 & N11MD2224 & 2012 & 13.2 & 13.1 & 2.1 & 1.3 \\
\hline B & NX1OMD 2216 & McGill a & 2012 & 13.2 & 14.4 & 2.1 & 1.8 \\
\hline C & NX1OMD 2216 & Wesley & 2012 & 13.2 & 15.1 & 2.1 & 1.5 \\
\hline D & Mattern a & N11MD2130 & 2012 & 13.3 & 13.5 & 1.9 & 1.7 \\
\hline E & Mattern b & Jerry & 2011 & 13.7 & 16.5 & 1.8 & 1.5 \\
\hline F & NX10MD 2300 & McGill b & 2012 & 14.9 & 13.7 & 2.1 & 1.6 \\
\hline G & NX10MD 2300 & N11MD2182 & 2012 & 14.9 & 14.6 & 2.1 & 1.8 \\
\hline H & NX1OMD 2300 & Mace & 2012 & 14.9 & 15.6 & 2.1 & 1.4 \\
\hline
\end{tabular}

ling. For each pair, 29 mixture samples were created, each $50 \mathrm{~g}$ in mass, of the following weight concentrations (conventional to total weight), in percent: 0, 1, 2, 3, 4, 5, 10, 15, ... 95, 96, 97, 98, 99, 100. Each test sample was thoroughly mixed by hand-tumbling of the material contained in an oversized plastic vial. Approximately half of each test sample was milled during an earlier study, ${ }^{15}$ and therefore not available for the current study. From the remaining material in each sample, $20 \mathrm{~g}$ was used in the HSI data collection.

\section{Equipment}

The HSI system, assembled in the USDA Beltsville laboratory, consisted of an InGaAS focal-plane array camera of dimensions $320 \times 256$ pixels and a 14-bit A/D (Xenics, Model Xeva-1.7-320, Leuven, Belgium), an imaging spectrograph (SWIR Hyperspec, Headwall Photonics, Fitchburg, MA, USA), two low-OH fibre optic bundles that conveyed light from separate DC-regulated $150 \mathrm{~W}$ quartz tungsten halogen light sources (Dolan Jenner, Model DC-950, Boxborough, MA, USA) to the imaging enclosure and a stepper motor movable stage (Velmex, Model XN10-0180-M02-21, Bloomfield, NY, USA) that moved a frame (described below) containing the kernels in a direction perpendicular to the camera's line field of view. The termini of the fibre bundles were single fibres arranged in $250 \mathrm{~mm}$ lines (overlapping the width of the line image field of view), with the two lines oppositely oriented at $30^{\circ}$ with respect to the vertical and parallel to the line field of view. A 25-mm zoom lens (Optec, Model OB-SWIR25/2, Parabiago, Italy) was connected to the front end of the spectrograph. With a working distance of $430 \mathrm{~mm}$ (front of lens to object surface), the actual line field of view was $180 \mathrm{~mm}$. To maintain square pixels, the number of lines was set at 470 and the distance between lines was set at $0.56 \mathrm{~mm}$. The $20 \mathrm{~g}$ of kernels of each test sample, typically about 700 kernels, were spread over a $165 \mathrm{~mm} \times 253 \mathrm{~mm}$ rectangular region in a plywood frame lined with black course grit emery cloth (3M). Kernels were manually spread apart to minimise touching occurrences. During scanning, the frame was momentarily stopped during collection of a line spectrum, after which the stage was advanced the incremental distance before collection of the next line. The effective wavelength range was $940-1650 \mathrm{~nm}$, and with 150 spectral bands collected, the average spacing between bands was $4.7 \mathrm{~nm}$. Spectral data was stored in two-byte integer format in a hypercube of dimensions $320 \times 470 \times 150$. Details on the spectral and spatial calibration of the system are described elsewhere. ${ }^{22}$

In this experiment, a dark current image was collected on an hourly basis (approximately after every four samples) by covering the camera lens. Likewise, a 99\% reflectance standard, consisting of sintered PTFE (Spectralon ${ }^{\mathrm{TM}}$, SRT-99-120, Labsphere, North Sutton, NH, USA), was collected immediately after the dark current image. These two images were subsequently applied to the sample images collected before the next pair of dark current and "white standard" images.

\section{Image processing}

Data reduction and spectral analysis tasks were performed in MATLAB (v. 2016a) equipped with MATLAB's image processing toolbox. The intensity readings of each test sample's hypercube were transformed to reflectance by dividing the dark current-subtracted intensity by the dark current-subtracted white standard intensity at each of the corresponding spectral bands (i.e., image slices). For removing the emery cloth background, all pixels having reflectance at the tenth band $(981 \mathrm{~nm})$ less than 0.3 were 
set to 0 , and the remaining pixels were set to 1 . This mask was then applied to all slices in the sample's hypercube. Using the tenth band, object (kernel) boundaries were first eroded using a disk-shaped structuring element of radius 1 to remove pixels along the contour for the reason that these would otherwise contribute low reflectance spectra due to the curvature of the kernel. Following erosion, relevant objects were identified as contiguous regions of five or more pixels. Smaller regions were discarded. Objects of greater than 35 pixels, indicating two or more touching kernels, were likewise discarded. The toolbox functions imerode and bwareaopen were used in these respective erosion and object identification steps. Comparisons of the numbers of kernels identified in image processing and human visual counts, performed for all of the pure waxy and pure conventional samples, indicated close agreement, typically within $2 \%$ of the actual number. Altogether, the eight pairs, at 29 test samples per pair, produced 232 test samples for imaging.

\section{Spectral analysis}

Each identified object from image processing, hereafter termed a kernel, was reduced to a mean reflectance spectrum by averaging over all pixels within the object. Noisy conditions at the longest wavelengths led to the dropping of five bands at the right end of the spectrum, thus leaving 145 "bands" spanning 938-1630 nm. Partial least squares (PLS) regression was applied in the data analysis using the PLS function and various preprocessing functions available in PLS Toolbox 7.3 (Eigenvector Research, Wenatchee, WA, USA) operating in MATLAB. Preprocessing usually consisted of a standard normal variate (SNV) transformation ${ }^{23}$ followed by a Savitsky-Golay convolution of either smooth, first derivative or second derivative, always using a second order polynomial approximation. ${ }^{24}$ Preliminary trial and error operations resulted in the choice of a seven-point window for all convolutions. The SNV step was necessary to compensate for large differences in overall reflectance among kernels irrespective of their conventional or waxy pedigree, as caused by variation in size and shape. The exception to these preprocessing steps occurred when statistical pattern recognition classifiers were implemented as the third of three approaches, in which case the reflectance spectra were directly used as input features.

Using the preprocessed mean kernel spectra, PLS regression models were developed in two of the three approaches. In the first approach, a PLS calibration equation was formed by using the pure conventional and pure waxy test samples from seven of the eight pairs. Upon randomly selecting 300 conventional kernels and an equal number of waxy kernels from each pair, a PLS regression calibration was developed, with 0 and 1 , respectively, assigned to waxy and conventional kernels. By trial and error, the number of PLS factors that consistently produced stable results was determined to be seven. Hence, each seven-factor PLS calibration was applied to all test samples of the left-out pair using the midpoint (0.5) as the classification boundary between conventional and waxy for each kernel in a test sample. Counts of classified conventional and waxy kernels within a test sample were then used to estimate mixture level percentage $Y$,

$$
Y=100 \frac{\sum_{i=1}^{n_{c}} A_{i}^{3 / 2}}{\sum_{j=1}^{n_{c+w}} A_{j}^{3 / 2}}
$$

where $i$ is the summation index over the kernels classified as conventional $c, j$ is the summation index over all kernels $c+W$ and $A$ is the area of a kernel (object) in pixels. The 3/2 exponent was a simple attempt to transform the area of an object into a volume, and hence a representation of mass (assuming equal physical densities and shapes between conventional and waxy kernels). Once the model was applied to all test samples of the removed pair, a new PLS calibration was developed using a different set of seven pure conventional and seven pure waxy test samples and subsequently applied to all test samples of the newly left-out pair. This process was repeated until each pair was removed during calibration and used in validation. Reported model statistics, ${ }^{25}$ as calculated on each validated pair, included the coefficient of determination between actual and modelled mixture level $\left(r^{2}\right)$, bias and the standard error of prediction (SEP). Each figure of merit was eventually averaged over the eight pairs for tabulated presentation of results.

In the second approach to modelling, PLS regression calibrations were developed using all 29 test samples per pair, 7 pairs at a time $(n=203)$, with each test sample represented as one spectrum calculated as the mean of all kernel mean spectra within the test sample, and the dependent variable as the known mixture level for that sample. The calibration equation was applied to the 29 test samples of the left-out pair, and the calibration and validation cycle was then repeated with the interchanging of one of the seven pairs with the left-out pair, and so on, until each pair had its turn in model validation. 
Mixture level residuals were used to calculate the same set of statistics as in the first approach.

The third approach involved statistical pattern recognition classifier functions available in the MATLAB open source toolbox PRTools (v. 5.3.3, http://prtools.org), as described in the toolbox developer's book. ${ }^{26}$ PRTools is a statistical pattern recognition toolbox that provides many MATLAB-coded routines for classifier training and evaluation, feature selection and extraction, and data and result visualisation. Five classifiers in the PRTools were used, including stats linear classifier (statslinc), Fisher's linear classifier (fisherc), k-nearest neighbour classifier (knnc), decision tree classifier (dtc) and nearest mean classifier (nmc). The five classifiers were trained using the original mean reflectance spectra from seven of the eight pairs of all pure conventional kernels with one label and pure waxy kernels with a second label. Adjustable parameters were automatically set to default values during model training. Each trained classifier was then evaluated by all the samples in the left-out pair, with the midpoint (0.5) used as the classification boundary between the conventional and waxy kernels. Tallies of kernels weighted by area raised to the three halves power in the two classes were used to determine the conventional weight percentage in the same fashion as the first approach.

An ANOVA (Proc MIXED, SAS Institute, Cary, NC, USA) was performed on the three pre-averaged figures of merit to evaluate the effect of model type and preprocess or classifier.

\section{Ground truth image}

For one test sample, upon completing the HSI scanning, a slab of white polymer modelling clay (Original Sculpey ${ }^{\top M}$ ), rolled out to the dimension of the scan tray, was placed over the kernels and pressed with sufficient pressure to embed the kernels in the clay. The slab was cured at $130^{\circ} \mathrm{C}$ for $20 \mathrm{~min}$ and sanded flat with 100 -grit sandpaper to expose kernel endosperm. An iodine solution (0.1\% $w / v)$ was applied to the surface of the slab, whereupon conventional kernels were identified by their purple colour.

\section{Results and discussion}

The spectral similarity between conventional and waxy kernels is shown through the series of preprocess transformations in Figure 1. The upper graph (A) contains the grand means of the eight pure conventional and

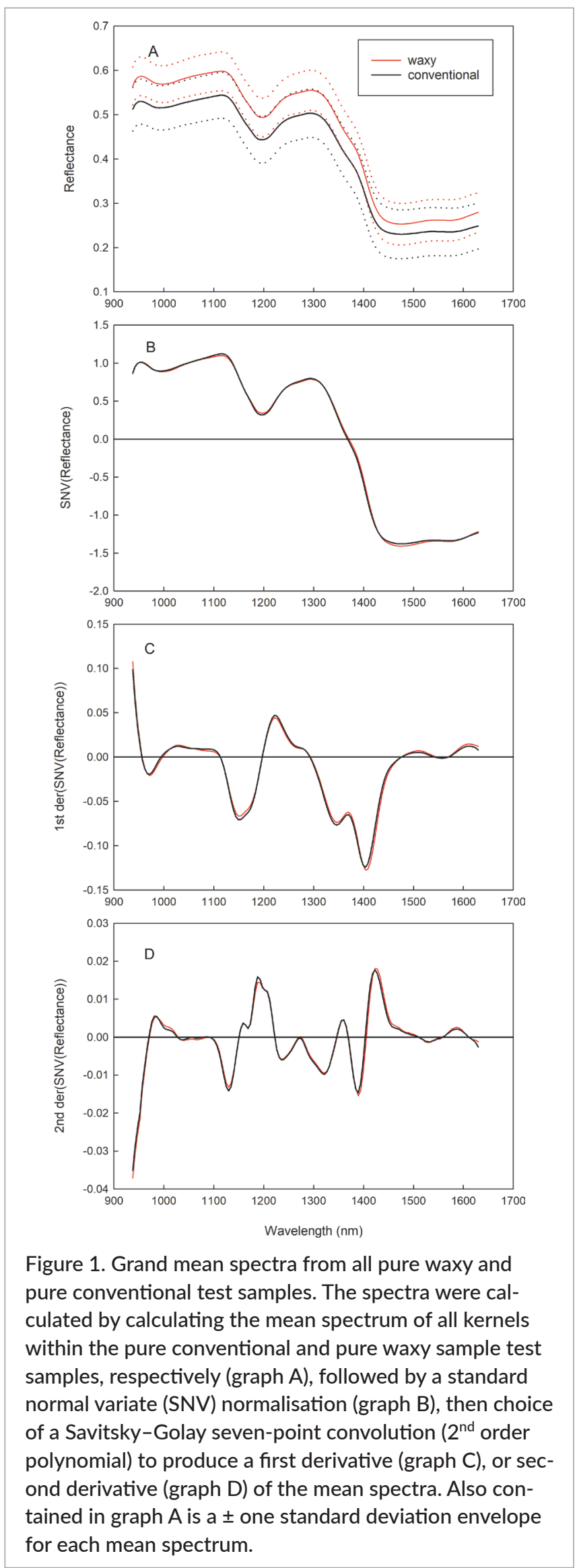


eight pure waxy test sample spectra, in which each test sample's mean spectrum was calculated as the average of approximately 700 individual kernel mean spectra. A one standard deviation envelope is also shown for each grand mean spectrum. The broadness of the envelopes alludes to a possible difficulty in classifying individual kernels based on raw reflectance. The authors' earlier study using diffuse reflectance spectroscopy (1100-2500 nm) of ground meal demonstrated the advantage of spectral preprocessing to reduce physical effects, especially with respect to the wavelength region beyond $2000 \mathrm{~nm} .{ }^{15}$ Therefore, preprocessing was performed on the current study's HSI data, albeit over the narrower wavelength region. With removal of most of the differences attributed to physical effects (e.g., size, shape, texture), the transformed spectra became nearly identical in appearance after successive applications of SNV normalisation (Figure 1B) and first derivative transformation (Figure 1C) or second derivative transformation (Figure 1D).

The means of the statistical figures of merit for the two PLS approaches and the pattern recognition approach are summarised in Table 2. For the first PLS approach (percentages of weighted classified kernel counts, hereafter called the PLSDA approach), the mean $r^{2}$ values were $0.932,0.941$ and 0.873 for smooth, first derivative and second derivative preprocesses, respectively. The corresponding mean of absolute bias were 5.04\%, 4.32\% and $5.94 \%$, respectively, while SEPs were 10.3\%, 9.6\% and $13.6 \%$, respectively. With the first derivative preprocess demonstrating the best performance among the PLSDA models, plots of actual versus predicted mixture levels based on this approach are shown in Figure $2 \mathrm{~A}-\mathrm{H}$, with each plot representing a validated pair. Some pairs, such as $E(S E P=4.0 \%)$, demonstrated close agreement between actual and predicted values, while other pairs, such as A (SEP $=16.5 \%)$ were more difficult to model. In general, samples possessing higher conventional fractions had larger deviations between measured and modelled percentages than samples possessing lower fractions.

The corresponding actual versus predicted plots for the second PLS approach (regression on mean spectra, hereafter called the PLS1 approach) with first derivative preprocessing (again, the best of the three preprocesses) are shown in Figure 3A-H. Compared to the PLSDA approach, the SEPs among the validation pairs from the PLS1 approach were more similar to one another, with the best pair $B(S E P=6.4 \%)$ and worst pair $E(S E P=11.6 \%)$ indicating a smaller overall range. As with the PLSDA approach, deviations between measured and modelled values increased as the conventional percentage increased. The disadvantage of the PLS1 approach is that through spectral averaging the potential benefit of imaging is lost because kernels are not individually classified and therefore not capable of being sorted.

For the pattern recognition classifier models (Table 2), two of the classifiers, statslinc and fisherc, with

Table 2. Hyperspectral imaging model statistics for validation set. Values are the means of the statistics of eight pairs.

\begin{tabular}{|l|l|l|l|l|}
\hline \multirow{4}{*}{ Model type } & $\begin{array}{l}\text { Preprocess or } \\
\text { classifier }^{\text {a }}\end{array}$ & $r^{2}$ & $\mid$ Bias| (percentage units) & SEP (percentage units) \\
\hline \multirow{4}{*}{ PLSDA } & smooth & $0.932 \mathrm{a}$ & $5.04 \mathrm{abcd}$ & $10.3 \mathrm{a}$ \\
\cline { 2 - 5 } & $1^{\text {st }}$ derivative & $0.941 \mathrm{a}$ & $4.32 \mathrm{abcd}$ & $9.6 \mathrm{a}$ \\
\cline { 2 - 5 } & $2^{\text {nd }}$ derivative & $0.873 \mathrm{bc}$ & $5.94 \mathrm{bcde}$ & $13.6 \mathrm{~b}$ \\
\hline \multirow{4}{*}{ PLS1 } & smooth & $0.953 \mathrm{a}$ & $6.65 \mathrm{de}$ & $9.4 \mathrm{a}$ \\
\cline { 2 - 5 } & $1^{\text {st }}$ derivative & $0.959 \mathrm{a}$ & $6.54 \mathrm{cde}$ & $9.4 \mathrm{a}$ \\
\cline { 2 - 5 } & $2^{\text {nd }}$ derivative & $0.952 \mathrm{a}$ & $8.19 \mathrm{e}$ & $9.5 \mathrm{a}$ \\
\hline \multirow{4}{*}{ Pattern } & statslinc & $0.949 \mathrm{a}$ & $2.74 \mathrm{a}$ & $8.9 \mathrm{a}$ \\
\cline { 2 - 5 } & fisherc & $0.946 \mathrm{a}$ & $3.33 \mathrm{ab}$ & $9.1 \mathrm{a}$ \\
\cline { 2 - 5 } & knnc & $0.910 \mathrm{ab}$ & $4.90 \mathrm{abcd}$ & $17.9 \mathrm{c}$ \\
\cline { 2 - 5 } & $\mathrm{dtc}$ & $0.921 \mathrm{a}$ & $3.61 \mathrm{abc}$ & $18.3 \mathrm{c}$ \\
\cline { 2 - 5 } & $\mathrm{nmc}$ & $0.830 \mathrm{c}$ & $5.64 \mathrm{abcde}$ & $22.0 \mathrm{~d}$ \\
\hline
\end{tabular}

${ }^{a}$ Smooth and derivative preprocesses included a preliminary step of a standard normal variate (SNV) transformation. ${ }^{23} \mathrm{~A}$ smooth or derivative was performed by Savitzky-Golay seven-point convolution using a second order polynomial. Classifier abbreviations: statslinc = stats linear, fisherc $=$ Fisher's linear, $\mathrm{knnc}=k$-nearest neighbour, $\mathrm{dtc}=$ decision tree, $n \mathrm{nmc}=$ nearest mean .

Within a column, pretreatments are significantly different for letters that are not in common $(p<0.05)$. 

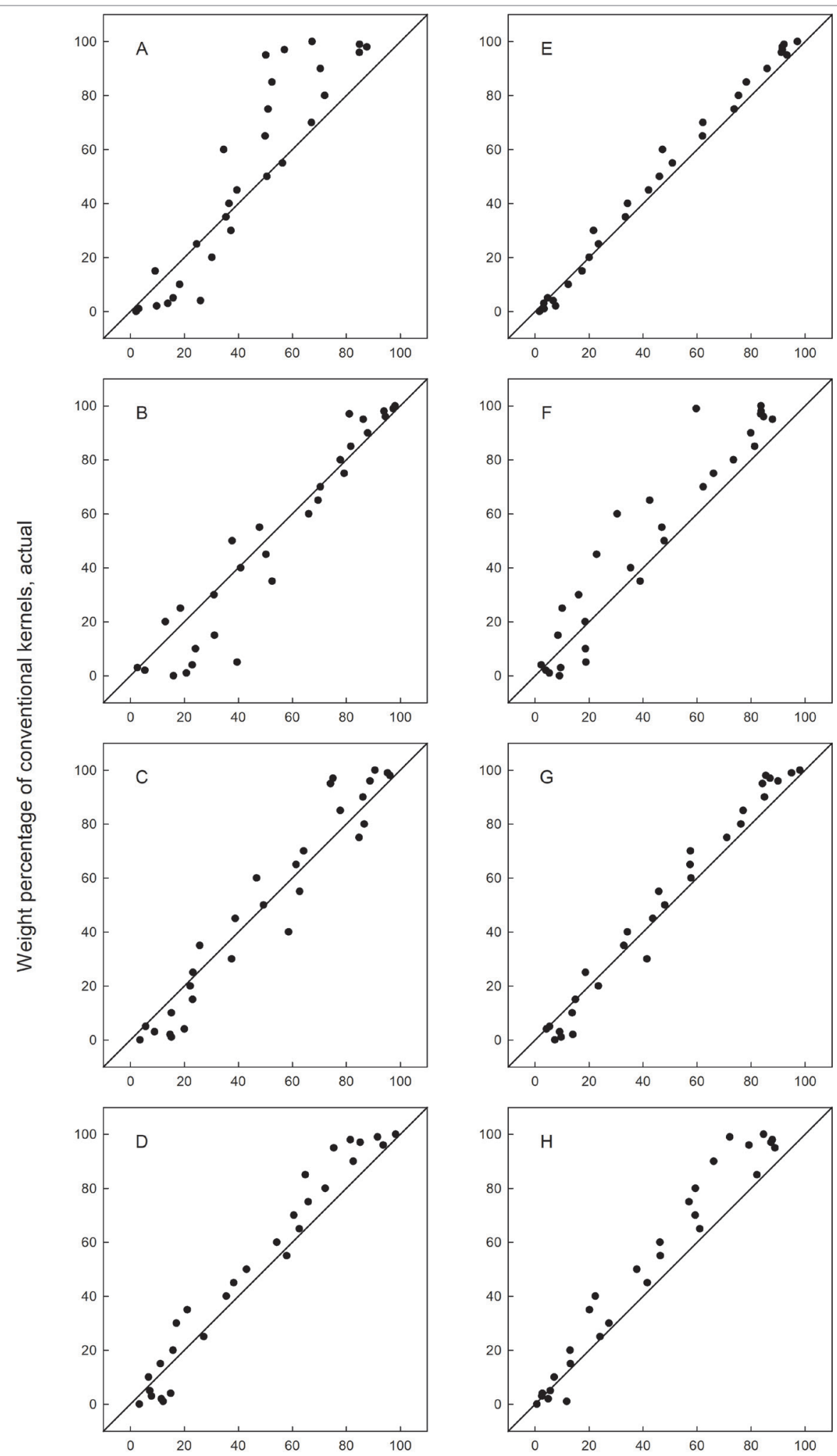

Weight percentage of conventional kernels, HSI PLSDA

Figure 2. Actual vs predicted scatter plots of each pair $(\mathrm{A}-\mathrm{H})$ as a validation set, based on two-class PLSDA to assign kernels into waxy or conventional classes, with calibration (seven-factor, first derivative after SNV) performed using pure waxy and pure conventional spectra from the seven other pairs, then counts of classified waxy and conventional kernels in validation test samples are tallied and percentages are calculated. Graphs A-H correspond to pairs A-H, respectively. 

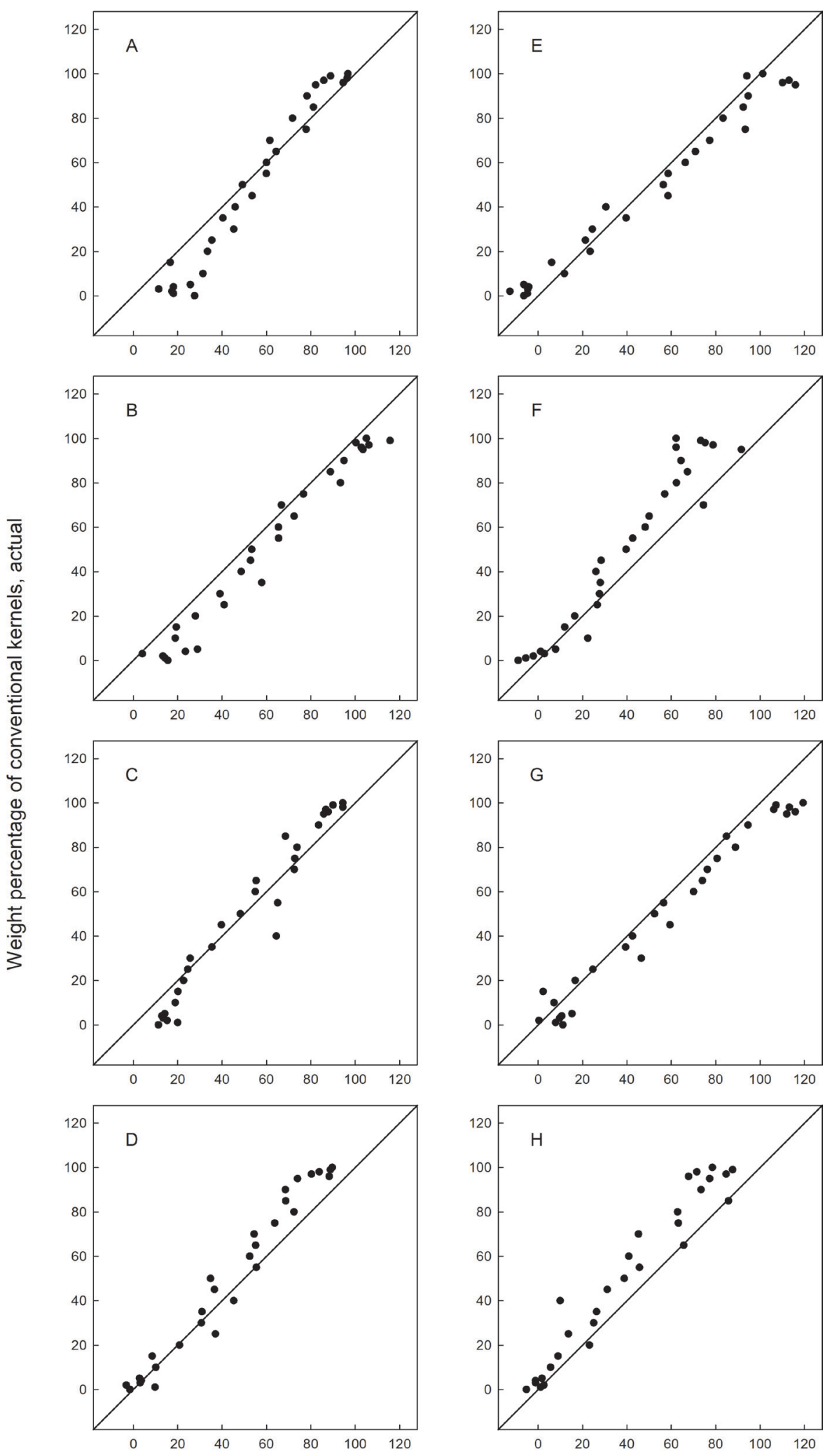

Weight percentage of conventional kernels, HSI PLS1

Figure 3. Actual vs predicted scatter plots of each pair $(\mathrm{A}-\mathrm{H})$ as a validation set, based on PLS1, with calibration (sevenfactor first derivative after SNV) performed on all test samples from the seven other pairs. Graphs A-H correspond to pairs A-H, respectively. 

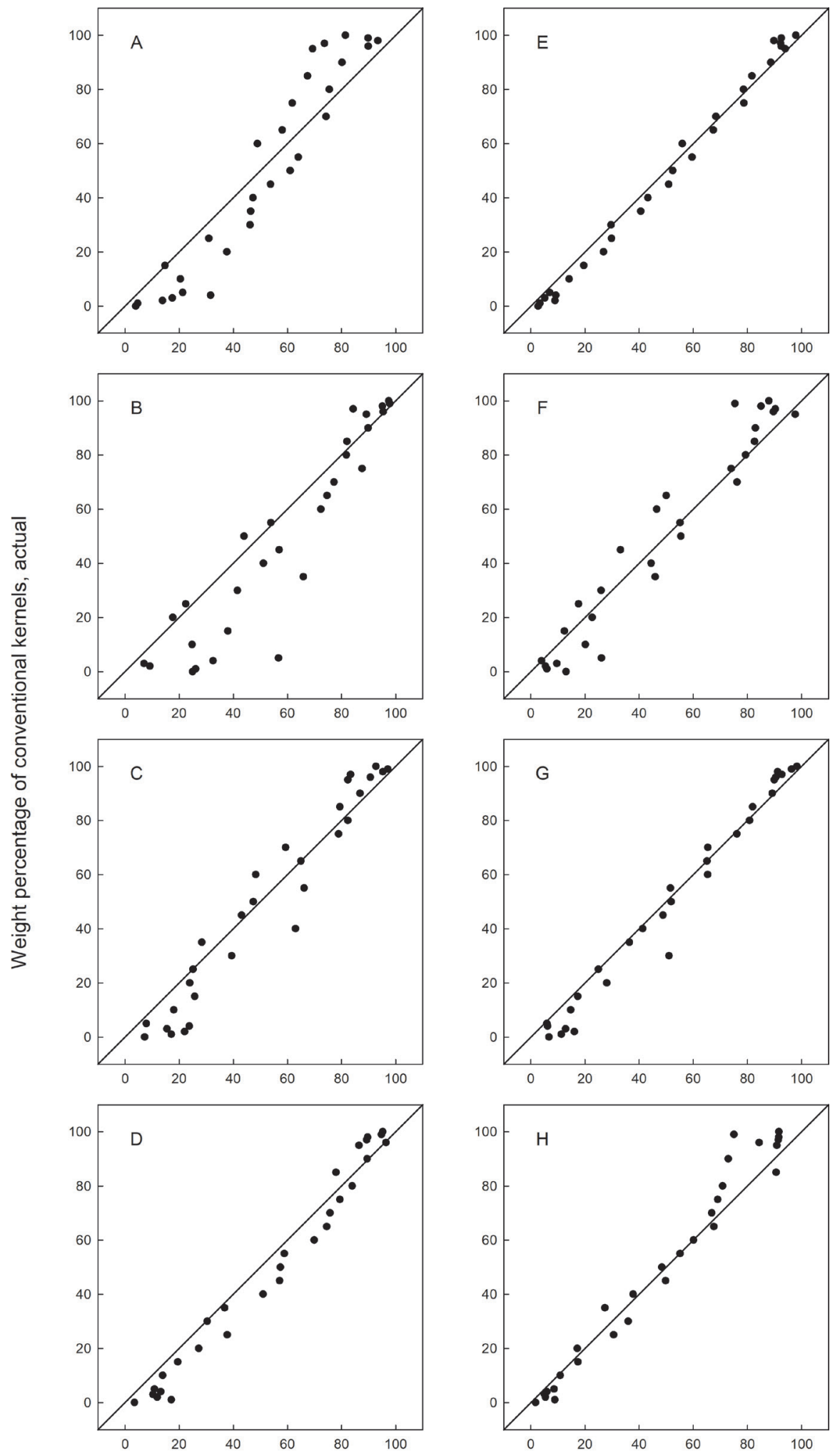

Weight percentage of conventional kernels, HSI statslinc

Figure 4. Actual vs predicted scatter plots of each pair $(\mathrm{A}-\mathrm{H})$ as a validation set, based on pattern recognition function statslinc, with calibration performed using the pure waxy and pure conventional spectra of all kernels from the seven other pairs, then counts of classified waxy and conventional kernels in validation test samples are tallied and percentages are calculated. Graphs A-H correspond to pairs A-H, respectively. 


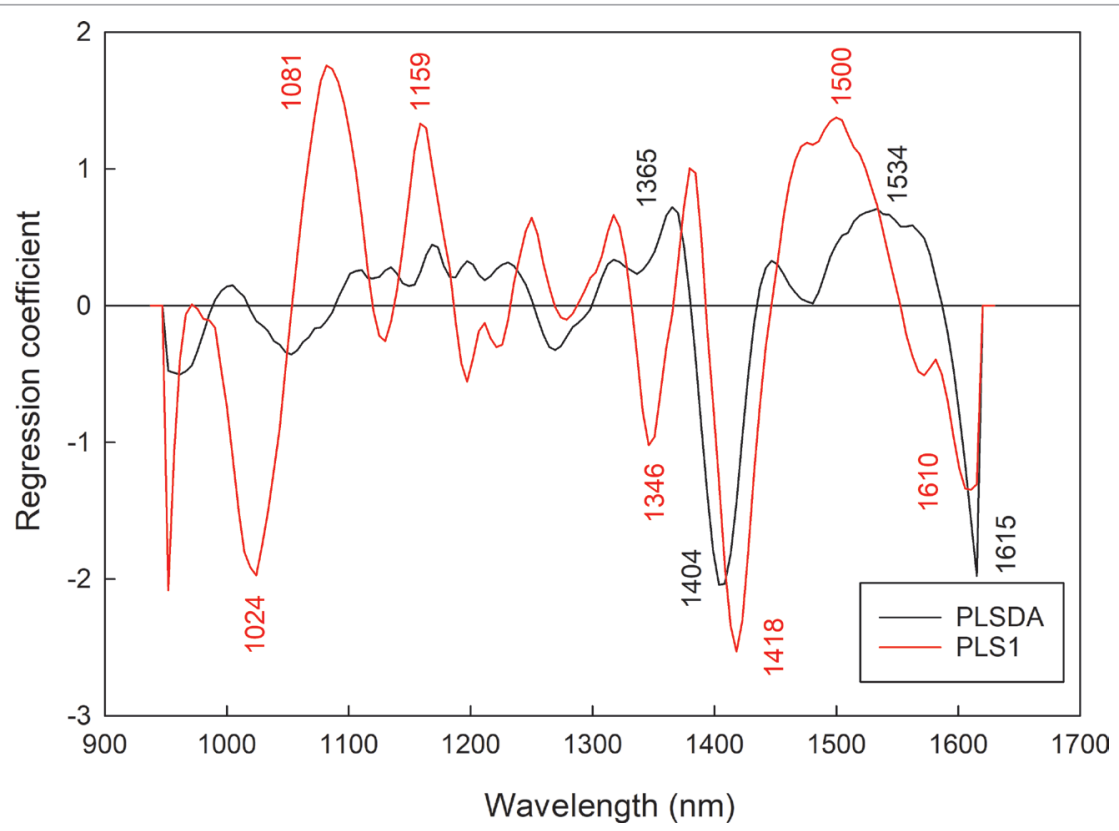

Figure 5. Regression vectors for PLSDA and PLS1 models. Models were both seven-factor on SNV followed by SavitskyGolay seven-point smoothed spectra.

respective SEPs of $8.9 \%$ and $9.1 \%$, had performances that were similar to the best of the PLSDA and PLS1 models. However, the other three classifiers (knnc, dtc and $\mathrm{nmc}$ ), with SEPs ranging between 17.9\% and 22.0\%, demonstrated significantly poorer performance. Actual versus modelled mixture level plots for the eight pairs are shown for the statslinc classifier in Figure 4A-H. The plots are closer in appearance to the corresponding PLSDA plots in Figure 2 than they are to the ones of the PLS1 model in Figure 3. The closer similarity is most likely because the PLSDA and pattern recognition approaches were based on counts of kernels weighted by their areas, as opposed to the PLS1 approach which was based on conventional quantitative modelling of mean spectra. Although it produced similar results to PLSDA, the pattern recognition approach is not readily adaptable to spectral interpretation.

Using the calibration set that excluded pair $E$ by example, regression vectors for the PLSDA and PLS1 calibrations are shown in Figure 5. For both approaches, preprocessing consisted of SNV followed by a SavitskyGolay seven-point smooth. The most striking feature of this graph is that the approaches produced different local maxima and minima, with wavelengths of 1365, 1404, 1534 and $1615 \mathrm{~nm}$ for the PLSDA model and 1024 , 1081, 1159, 1346, 1418, 1500 and $1610 \mathrm{~nm}$ for the PLS1 model. Coincidently, the wavelengths with largest absolute coefficients for the PLSDA and PLS1 models, at $1404 \mathrm{~nm}$ and $1418 \mathrm{~nm}$, respectively, fell within the range of 1350-1450 nm that the authors had previously identified as most important in bulk whole grain analysis of conventional and waxy mixtures. ${ }^{15}$

Calibration and validation sets were purposely structured to be identical to those of previous research that utilised conventional NIR spectroscopy ${ }^{15}$ to facilitate the direct comparison between HSI and the conventional approach. In the current study, a new analysis was also performed to determine whether the validation statistics were being falsely inflated because of occurrences when the waxy parent sample was present in both calibration and validation sets. (As shown in Table 1, pairs $A-C$ had the same waxy parent sample, as did pairs $\mathrm{F}-\mathrm{H}$.) For the new analysis, calibration and validation sets were structured so as not to have any parent waxy sample common to both, thus potentially affecting the validation statistics on six of the original eight pairs. The results indicated non-significant differences in the PLSDA statistics between the regular and new analyses. For example, the mean values for SEP, |Bias $\mid$ and $r^{2}$ in the new analysis were 9.8\%, 3.81\% and 0.937, respectively, for the $1^{\text {st }}$ derivative preprocess using the same number of PLS factors (seven) as the original PLSDA. Likewise, non-significant differences in the statistics from the PLS1 model approach were found between regular and new analyses, though fewer factors (five) were needed in the new analysis. 


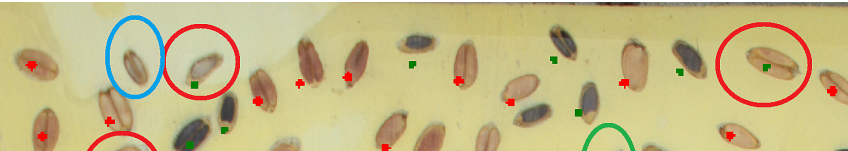

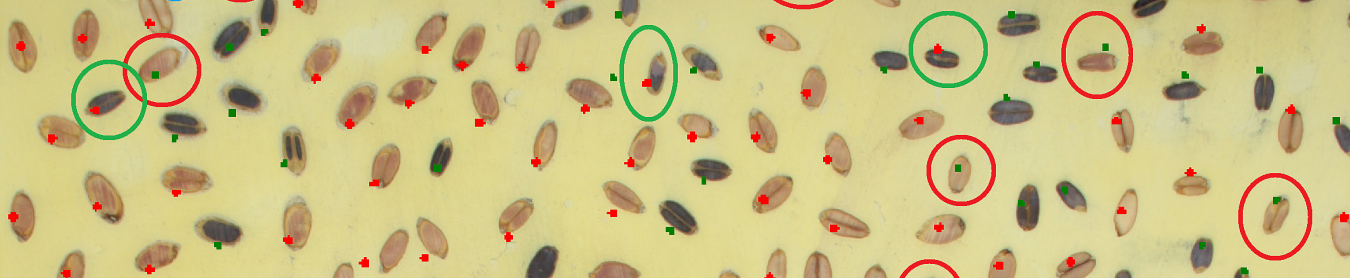
$\because 000=0000000000$

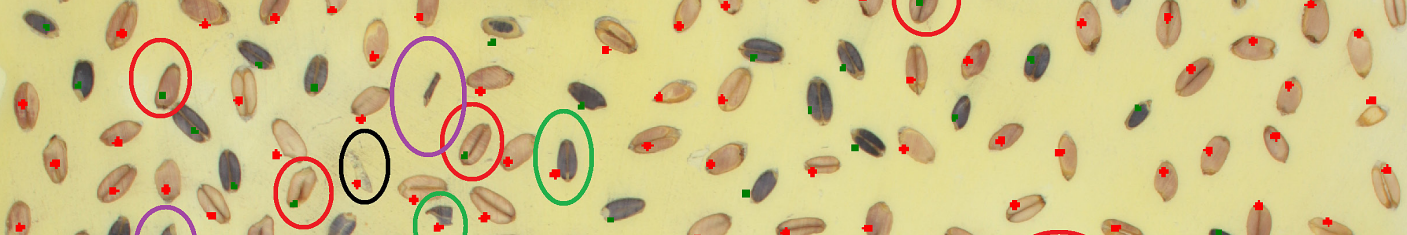

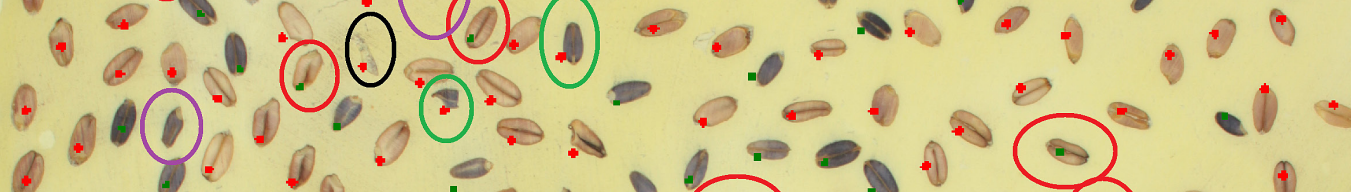
$0.00=1,0=100010$

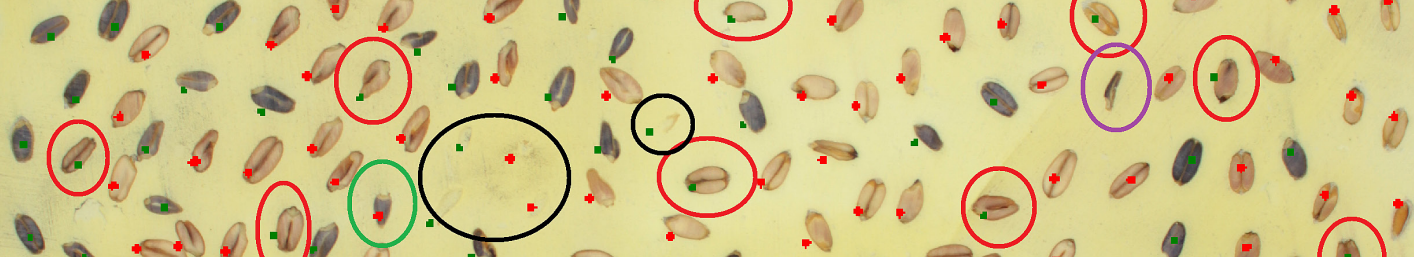

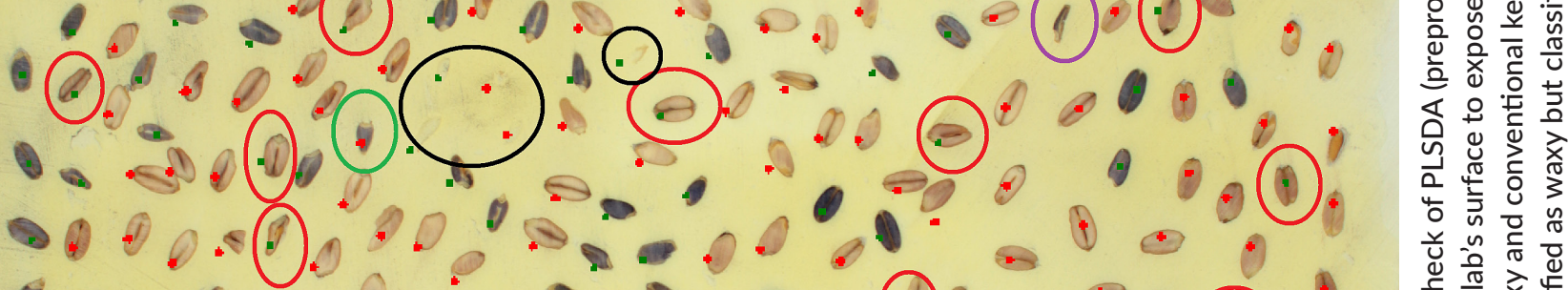

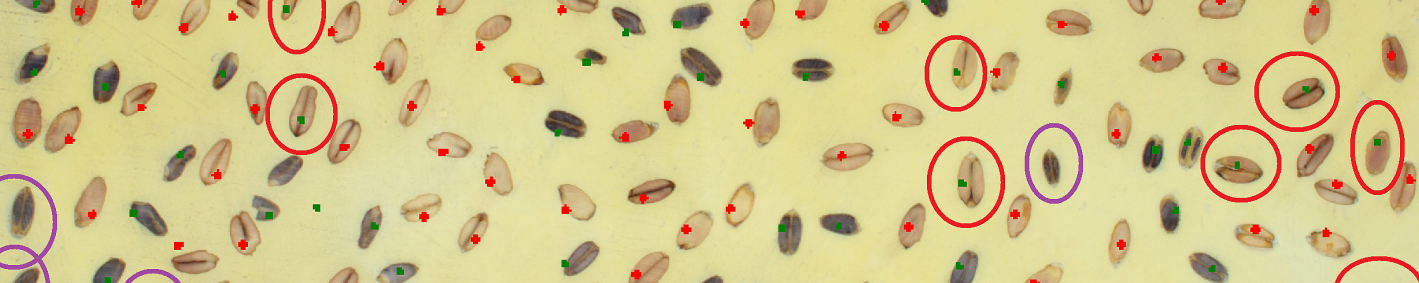
$0.0=0.000 .0 .0$ $\because 000000000$ $20=000000$

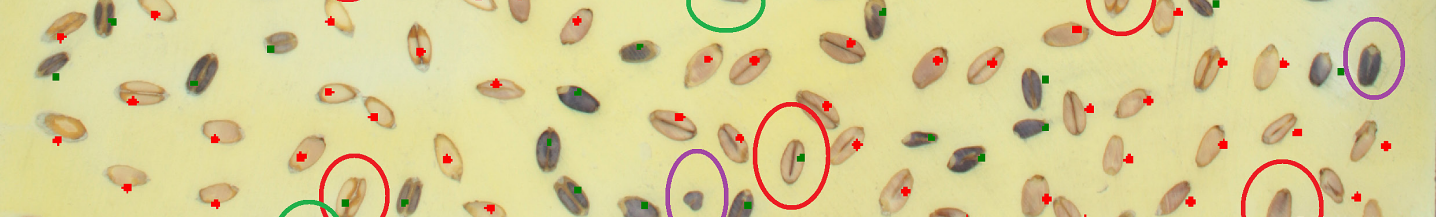

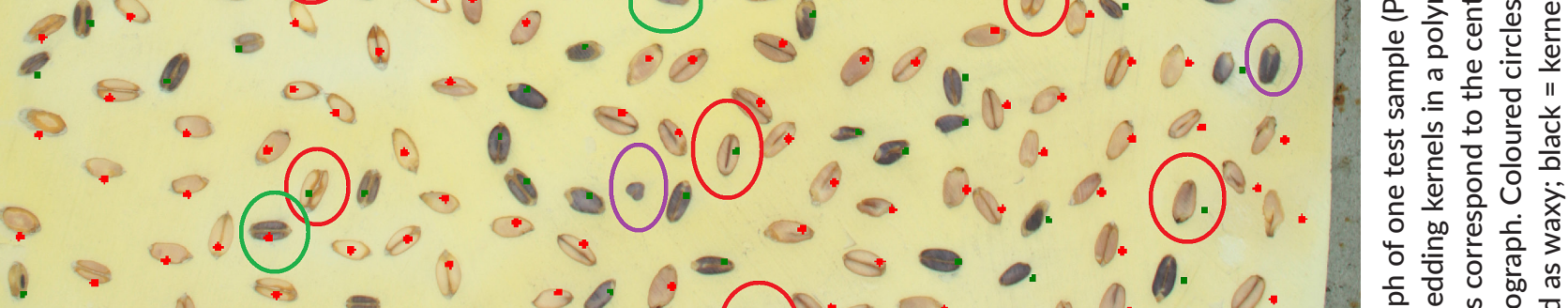

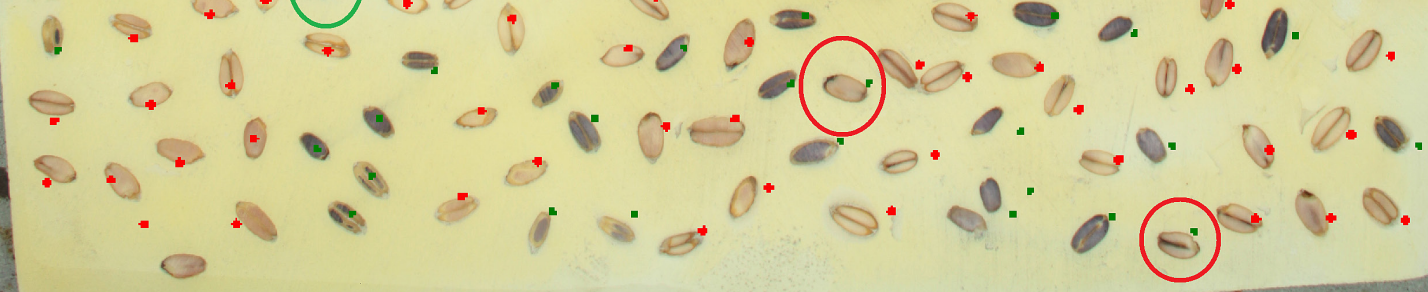


Lastly, the photograph of the sample used in ground truth verification is displayed in Figure 6. Dark coloured kernels, whose darkness arose from amylose reacting with iodine to form a blue colour complex, were counted to be 205. This is compared to 225 kernels identified as conventional by the PLSDA on first-derivative-of-SNV model. For the waxy condition, 435 and 402 kernels were identified by the visual and HSI methods, respectively. Disagreements between visual and HSI are shown as green circles ( 9 conventional kernels classified as waxy) and red circles (40 waxy kernels classified as conventional). The difference in total count between visual and HSI analyses, at 13 kernels, was caused by two conditions: the removal during image processing of oversized objects arising from touching kernels, thus depressing the HSI counts, and, second, a small number of kernels lost during the sanding operation (black circles in photograph).

\section{Conclusions}

Although spectral differences between conventional and waxy wheat are slight, NIR hyperspectral imaging of intact kernels may be used to measure mixture levels (w conventional / w total), with standard errors typically in the range of 9-13 percentage units. PLSDA and statistical pattern recognition approaches produced similar error rates. Although model performance was slightly less than that achieved by conventional NIR reflectance spectroscopy of bulk whole kernels or ground meal, the $\mathrm{HSI}$ technique offers the potential advantage of being adapted to sorting operations.

\section{References}

1. T. Nakamura, M. Yamamori, H. Hirano, S. Hidaka and T. Nagamine, "Production of waxy (amylose-free) wheats", Mol. Gen. Genet. 248, 253-259 (1995). doi: https://doi.org/10.1007/BF02191591

2. R.A. Graybosch, P.S. Baenziger, D.K Santra, T. Regassa, Y. Jin, J. Kolmer, S. Wegulo, G. Bai, P. St. Amand, X. Chen, B. Seabourn, F. Dowell, R. Bowden and D.M. Marshall, "Registration of 'Mattern' waxy (amylose-free) winter wheat", J. Plant Regist. 8, 43-48 (2014). doi: https://doi.org/10.3198/ jpr2013.08.0045crc
3. R.A. Graybosch, J.B. Ohm and L. Dykes, "Observations on the quality characteristics of waxy (amylose-free) winter wheats", Cereal Chem. 93, 599-604 (2016). doi: https://doi.org/10.1094/ CCHEM-02-16-0021-R

4. R.A. Graybosch, "Waxy wheats: Origin, properties, and prospects", Trends Food Sci. Technol. 9, 135-142 (1998). doi: https://doi.org/10.1016/S09242244(98)00034-X

5. P.V. Hung, T. Maeda and N. Morita, "Waxy and highamylose wheat starches and flours-Characteristics, functionality and application", Trends Food Sci. Technol. 17, 448-456 (2006). doi: https://doi. org/10.1016/j.tifs.2005.12.006

6. R. Zhao, X. Wu, B.W. Seabourn, S.R. Bean, L. Guan, L. Shi, J.D. Wilson, R. Madl and D. Wang, "Comparison of waxy vs. nonwaxy wheats in fuel ethanol fermentation", Cereal Chem. 86, 145-156 (2009). doi: https://doi.org/10.1094/CCHEM-86-20145

7. T. Nakamura, P. Vrinten, M. Saito and M. Konda, "Rapid classification of partial waxy wheats using PCR-based markers", Genome 45, 1150-1156 (2002). doi: https://doi.org/10.1139/g02-090

8. X.C. Zhao and P.J. Sharp, "An improved 1-D SDSPAGE method for the identification of three bread wheat 'waxy' proteins", J. Cereal Sci. 23, 191-193 (1996). doi: https://doi.org/10.1006/jcrs.1996.0019

9. R.A. Graybosch, C.J. Peterson, L.E. Hansen, S. Rahman, A. Hill and J.H. Skerritt, "Identification and characterization of U.S. Wheats carrying null alleles at the wx loci", Cereal Chem. 75, 162-165 (1998). doi: https://doi.org/10.1094/ CCHEM.1998.75.1.162

10. S. Rahman, B. Kosarhashemi, M.S. Samuel, A. Hill, D.C. Abbott, J.H. Skerritt, J. Preiss, R. Appels and M.K. Morell, "The major proteins of wheat endosperm starch granules", Aust. J. Plant Physiol. 22, 793-803 (1995). doi: https://doi.org/10.1071/ PP9950793

11. J.F. Pedersen, S.R. Bean, D.L. Funnell and R.A. Graybosch, "Rapid iodine staining techniques for identifying the waxy phenotype in sorghum grain and waxy genotype in sorghum pollen", Crop Sci. 44, 764-767 (2004). doi: https://doi. org/10.2135/cropsci2004.7640

12. S.R. Delwiche and R.A. Graybosch, "Identification of waxy wheat by near-infrared reflectance spectros- 
copy", J. Cereal Sci. 35, 29-38 (2002). doi: https://doi. org/10.1006/jcrs.2001.0400

13. F.E. Dowell, E.B. Maghirang, R.A. Graybosch, W.A. Berzonsky and S.R. Delwiche, "Selecting and sorting waxy wheat kernels using near-infrared spectroscopy", Cereal Chem. 86, 251-255 (2009). doi: https://doi.org/10.1094/CCHEM-86-3-0251

14. S.R. Delwiche, R.A. Graybosch, P. St. Amand and G. Bai, "Starch waxiness in hexaploid wheat (Triticum aestivum L.) by NIR reflectance spectroscopy", J. Agric. Food Chem. 59, 4002-4008 (2011). doi: https://doi.org/10.1021/jf104528x

15. S.R. Delwiche and R.A. Graybosch, "Measurement of blend concentration of conventional and waxy hard wheats using NIR spectroscopy", Cereal Chem. 91, 358-365 (2014). doi: https://doi.org/10.1094/ CCHEM-09-13-0195-R

16. S.R. Delwiche and R.A. Graybosch, "Binary mixtures of waxy wheat and conventional wheat as measured by NIR reflectance", Talanta 146, 496-506 (2016). doi: https://doi.org/10.1016/j.talanta.2015.08.063

17. R.P. Cogdill, C.R. Hurburgh, G.R. Rippke, S.J. Bajic, R.W. Jones, J.F. McClelland, T.C. Jensen and J. Liu, "Single-kernel maize analysis by near-infrared hyperspectral imaging", Trans. ASAE 47, 311-320 (2004). doi: https://doi.org/10.13031/2013.15856

18. P. Williams, P. Geladi, G. Fox and M. Manley, "Maize kernel hardness classification by near infrared (NIR) hyperspectral imaging and multivariate data analysis", Anal. Chim. Acta 653, 121-130 (2009). doi: https://doi.org/10.1016/j.aca.2009.09.005

19. X. Yang, H. Hong, Z. You and F. Cheng, "Spectral and image integrated analysis of hyperspectral data for waxy corn seed variety classification", Sensors 15(7), 15578-15594 (2015). doi: https://doi. org/10.3390/s150715578
20.W.R. Morrison, T.P. Milligan and M.N. Azudin, "A relationship between the amylose and lipid contents of starches from diploid cereals", J. Cereal Sci. 2, 257-271 (1984). doi: https://doi.org/10.1016/S07335210(84)80014-4

21. S.H. Yoo and J.L. Jane, "Structural and physical characteristics of waxy and other wheat starches", Carbohyd. Polym. 49, 297-305 (2002). doi: https:// doi.org/10.1016/S0144-8617(01)00338-1

22. M.S. Kim, S.R. Delwiche, K. Chao, A. GarridoVaro, D. Perez-Marin, A.M. Lefcourt and D. Chan, "Visible to SWIR hyperspectral imaging for produce safety and quality evaluation", Sens. Instrum. Food Qual. Safety 5, 155-164 (2012). doi: https://doi. org/10.1007/s11694-012-9122-3

23. R.J. Barnes, M.S. Dhanoa and S.J. Lister, "Standard normal variate transformations and de-trending of near-infrared diffuse reflectance spectra", Appl. Spectrosc. 43, 772-777 (1989). doi: https://doi. org/10.1366/0003702894202201

24. A. Savitzky and M.J.E. Golay, "Smoothing and differentiation of data by simplified least squares procedures", Anal. Chem. 36, 1627-1639 (1964). doi: https://doi.org/10.1021/ac60214a047

25. T. Fearn, "Towards a standard terminology?", NIR news 15(4), 14-15 (2004). doi: https://doi. org/10.1255/nirn.779

26. F. van der Heijden, R.P.W. Duin, D. de Ridder and D.M.J. Tax, Introduction to PRTools. Classification, Parameter Estimation and State Estimation: An Engineering Approach Using MATLAB. Wiley, Chichester, pp. 405-415 (2004). doi: https://doi. org/10.1002/0470090154 